\title{
Repetibilidade de características agroindustriais em cana-de-açúcar
}

\author{
Maria Silvia Monteiro dos Santos ${ }^{(1)}$, José Antônio Madalena ${ }^{(1)}$, Lailton Soares ${ }^{(2)}$, Paulo Vanderlei Ferreira ${ }^{(2)}$ \\ e Geraldo Veríssimo Souza Barbosa(2)
}

\begin{abstract}
(1)Universidade Federal de Alagoas (Ufal), Centro de Ciências Agrárias (Ceca), Campus Delza Gitaí, BR 104 Norte, Km 85, CEP 57000-000 Rio Largo, AL. E-mail: maria.silvia.santos@bol.com.br, antoniomadalena@bol.com.br(2)Ufal, Ceca, Dep. de Fitotecnia e Fitossanidade. E-mail: lailtonsoares@bol.com.br, paulovanderleiferreira@bol.com.br, gvsb@fapeal.br
\end{abstract}

\begin{abstract}
Resumo - Os objetivos deste trabalho foram estabelecer estimativas de repetibilidade de características agroindustriais em 20 genótipos de cana-de-açúcar, determinar a previsibilidade de cada caráter e indicar a predição do valor verdadeiro de cada clone. O delineamento utilizado foi blocos casualizados, com cinco repetições. Utilizaram-se a análise de variância com dois fatores de variação (cortes e genótipos) e a análise dos componentes principais para estimar o coeficiente de repetibilidade $(\hat{\rho})$, a partir de três cortes. Foram obtidas estimativas de repetibilidade acima de 0,5 para fibra e toneladas de cana por hectare, em ambos os métodos, com confiabilidade maior que $84 \%$ pelo método dos componentes principais. As características que ficaram abaixo de 0,5, com previsibilidade inferior a 74\%, necessitam de um maior número de avaliações. Os métodos dos componentes principais e análise de variância indicaram, em cinco cortes, uma previsibilidade maior que $80 \%$ para fibra, porcentagem de pol (sacarose) no caldo da cana, toneladas de cana por hectare e toneladas de pol no caldo da cana por hectare, embora o primeiro tenha sido mais eficiente. Considerando toneladas de cana por hectare e toneladas de pol no caldo da cana por hectare, os clones RB9371, RB9350 e RB9364 são os melhores.
\end{abstract}

Termos para indexação: Saccharum, clones, seleção, análise de variância, componentes principais.

\section{Repeatability of agro-industrial characteristics in sugar cane}

\begin{abstract}
The objectives of this work were to establish repeatability estimates for agro-industrial characteristics for twenty genotypes of sugar cane, to indicate the determination coefficient of each character and the prediction of truthful value of each clone. The design consisted of randomized blocks, with five replications. The analysis of variance with two factors of variation (cuts and genotypes) and the analysis of the main components were used to estimate the repeatability coefficient $(\hat{\rho})$, initiating with three cuts. Estimates of repeatability were obtained over 0.5 for fiber and ton of cane per hectare, in both methods, with confidence over $84 \%$ by the method of main components. Characteristics lower than 0.5 and determination lower than $74 \%$ need a greater number of measures. The methods of main components and analysis of variance evidenced, in five cuts, determination over $80 \%$ for fiber, percentage of pol (sucrose) in the juice of cane, tons of cane per hectare and tons of pol in the juice of cane per hectare, though the first had been most efficient. Considering tons of cane per hectare and tons of pol in the juice of cane per hectare, the clones RB9371, RB9350 and RB9364 are the best.
\end{abstract}

Index terms: Saccharum, clones, selection, analysis of variance, principal components.

\section{Introdução}

Na região Nordeste do Brasil, a cana-de-açúcar é uma cultura de grande importância, tanto social como economicamente. Apesar de o Estado de Alagoas ser o segundo maior produtor de açúcar e álcool do país (Magalhães, 2000), os níveis de produtividade ainda são baixos, em virtude do acentuado déficit hídrico no período de setembro a fevereiro, dos solos pobres, e do uso de variedades com baixo potencial produtivo.
Segundo Barbosa et al. (2000), nas últimas três décadas, foi marcante a contribuição do melhoramento genético no desenvolvimento do setor canavieiro do Brasil, com ganhos acentuados de produtividade e qualidade. Nesse período, houve mais de $30 \%$ de aumento na média de produtividade da cana-de-açúcar e da recuperação de quilogramas de açúcar por tonelada de cana moída.

Ao escolher um genótipo, espera-se que sua superioridade inicial permaneça constante durante todo seu ci- 
clo. Segundo Cruz \& Regazzi (1997), também se espera que o bom desempenho de certas estruturas, ou de partes integrantes do indivíduo, reflita o potencial do genótipo a ser utilizado como um todo. Afirmam, ainda, que a veracidade desta expectativa poderá ser comprovada pelo coeficiente de repetibilidade da característica estudada e que é possível estimar este coeficiente quando a medição de um caráter é feita repetidas vezes num mesmo indivíduo.

O coeficiente de repetibilidade, segundo Vencovsky (1973), é utilizado no estudo de caracteres de plantas perenes que se expressam mais de uma vez no decorrer da vida do organismo. Baseia-se na tomada de mais de uma observação fenotípica de cada indivíduo, sem utilizar progênies, a fim de medir a capacidade que os organismos têm de repetir a expressão do caráter. De acordo com Falconer (1987), quando várias medidas de um mesmo caráter são feitas em cada indivíduo, a variância fenotípica poderá ser parcelada, servindo para quantificar o ganho em precisão, pela repetição das medidas, e esclarecer a natureza da variação causada pelo ambiente.

Adicionalmente, a repetibilidade fornece o valor máximo que a herdabilidade no sentido amplo pode atingir, pois expressa a proporção da variância fenotípica que é atribuída às diferenças genéticas confundidas com os efeitos permanentes que atuam na cultivar. Assim, esses parâmetros constituem ferramentas úteis para orientar os trabalhos de melhoramento (Botrel et al., 2000).

Estimativas de coeficientes de repetibilidade têm sido obtidas em culturas como seringueira (Gonçalves et al., 1982, 1990, 1995), coqueiro (Siqueira, 1982) e cupuaçuzeiro (Fonseca et al., 1990; Costa et al., 1997).

Os objetivos deste trabalho foram estabelecer estimativas de repetibilidade para as características agroindustriais em 20 clones de cana-de-açúcar, determinar a previsibilidade de cada caráter e indicar a predição do valor verdadeiro de cada clone.

\section{Material e Métodos}

O ensaio foi instalado em setembro de 1997 na Usina Caeté, localizada no Município de São Miguel dos Cam- pos, AL, subestação experimental do Programa de Melhoramento Genético de Cana-de-açúcar (PMGCA) do Centro de Ciências Agrárias (Ceca) da Universidade Federal de Alagoas (Ufal). O delineamento usado foi o de blocos casualizados, com cinco repetições. A unidade experimental era constituída por cinco fileiras de seis metros de comprimento distanciadas em 1,30 m. Os genótipos avaliados foram: RB72454, RB83102, SP791011 (padrões do PMGCA), RB937, RB9318, RB9326, RB9331, RB9333, RB9340, RB9348, RB9350, RB9353, RB9354, RB9358, RB9362, RB9364, RB9366, RB9367, RB9371 e RB9379. As colheitas foram realizadas no mês de novembro dos anos de 1998 (primeiro corte), 1999 (segundo corte) e 2000 (terceiro corte). Avaliaram-se cinco características agroindustriais da cana-de-açúcar: fibra, pureza, pol (porcentagem de sacarose aparente) no caldo da cana (PCC), tonelada de cana por hectare (TCH) e tonelada de pol (sacarose) no caldo da cana por hectare (TPH). Com base na média das cinco repetições de cada corte, obtiveram-se as estimativas do coeficiente de repetibilidade $(\hat{\rho})$, o coeficiente de determinação ou grau de previsibilidade de cada caráter $\left(\mathrm{R}^{2}\right)$, e a predição do valor verdadeiro de cada clone (PVVC) a partir de três cortes.

$\mathrm{Na}$ estimação do coeficiente de repetibilidade, foram utilizados dois métodos estatísticos, o método da análise da variância com dois fatores de variação (cortes e genótipos), e o método dos componentes principais.

No primeiro método, o modelo estatístico abaixo possibilita remover efeitos de ambiente temporário, de acordo com Cruz \& Regazzi (1994), em que:

$Y_{i j}=\mu+g_{i}+a_{j}+\varepsilon_{i j}$ sendo $Y_{i j}$ a observação referente ao i-ésimo genótipo (i $=1,2,3, \ldots, 20)$ e j-ésimo corte $(\mathrm{j}=1,2,3) ; \mu$, a média geral; $\mathrm{g}_{\mathrm{i}}$, o efeito aleatório do i-ésimo genótipo ( $\mathrm{i}=1,2,3, \ldots, 20) ; \mathrm{a}_{\mathrm{j}}$, o efeito fixo do $\mathrm{j}$-ésimo corte $(\mathrm{j}=1,2,3)$; $\varepsilon_{\mathrm{ij}}$, o erro experimental associado à observação $\mathrm{Y}_{\mathrm{ij}}$.

Por meio da análise de variância para o modelo utilizado, foram obtidos os quadrados médios (QM) e as esperanças dos quadrados médios [E(QM)], para genótipos e resíduo, respectivamente, dados por $\left(\sigma_{\mathrm{e}}^{2}+n \sigma_{\mathrm{g}}^{2}\right) \mathrm{e}\left(\sigma_{\mathrm{e}}^{2}\right)$. Deste modo, o coeficiente 
de repetibilidade é obtido por:

$\hat{\rho}_{(\mathrm{av})}=\frac{\operatorname{Côv}\left(\mathrm{Y}_{\mathrm{ij}}, \mathrm{Y}_{\mathrm{ij}}\right)}{\sqrt{\hat{\mathrm{v}}\left(\mathrm{Y}_{\mathrm{ij}}\right) \hat{\mathrm{v}}\left(\mathrm{Y}_{\mathrm{ij}}\right)}}=\frac{\hat{\sigma}_{\mathrm{g}}^{2}}{\hat{\sigma}_{\mathrm{y}}^{2}}=\frac{\hat{\sigma}_{\mathrm{g}}^{2}}{\hat{\sigma}_{\mathrm{e}}^{2}+\hat{\sigma}_{\mathrm{g}}^{2}}$,

em que $\hat{\rho}$ é a estimativa do coeficiente de repetibilidade; $\hat{\sigma}_{\mathrm{g}}^{2}$ é a estimativa da variância entre genótipos; $\hat{\sigma}_{\mathrm{e}}^{2}$ é a estimativa da variância ambiental; $\hat{\sigma}_{\mathrm{g}}^{2}=\frac{\mathrm{QMG}-\mathrm{QMR}}{\mathrm{n}}$; $\hat{\sigma}_{\mathrm{e}}^{2}=\mathrm{QMR}$. QMG é o quadrado médio de genótipos e QMR, o quadrado médio do resíduo.

O método dos componentes principais com base na matriz de correlações $(\mathrm{R})$ encontra-se descrito por Abeywardena (1972), Rutledge (1974) e Mansour et al. (1981). Consiste em se obter uma matriz de correlações (R) entre os genótipos em cada par de medições avaliadas $(\rho)$, dada por:

$\mathrm{R}=\left[\begin{array}{cccc}1 & \rho & \cdots & \rho \\ \rho & 1 & \cdots & \rho \\ \cdots & \cdots & & \cdots \\ \rho & \rho & & 1\end{array}\right]_{\mathrm{n}}$

Para a predição do valor verdadeiro do clone pela média de $\eta$ medidas repetidas, considerou-se $\bar{Y}_{i}$ como a média das $\eta$ avaliações sobre um caráter e $\overline{\mathrm{Y}}_{\infty}$ a média deste mesmo caráter, no mesmo clone, provenientes de infinitas avaliações. Neste caso, espera-se que $\bar{Y}_{\infty}$ seja igual ao valor real do clone, pois, em um número infinitamente grande de medições, os efeitos temporários do ambiente sobre o caráter tenderão a se cancelar. Deste modo, a correlação entre a média das observações entre $\eta$ medições com o valor real do clone pode ser obtida por meio de:

$\rho_{\infty}=\frac{\operatorname{Cov}\left(\overline{\mathrm{Y}}_{\mathrm{i}}, \overline{\mathrm{Y}}_{\infty}\right)}{\sqrt{\mathrm{V}\left(\overline{\mathrm{Y}}_{\mathrm{i}}\right) \cdot \mathrm{V}\left(\overline{\mathrm{Y}}_{\infty}\right)}}=\frac{\rho \mathrm{V}(\mathrm{Y})}{\sqrt{\frac{1+\rho(\eta-1)] \mathrm{V}(\mathrm{Y})}{\eta} \rho \mathrm{V}(\mathrm{Y})}}$ ou ainda: $\rho_{\infty}=\sqrt{\frac{\eta \rho}{1+\rho(\eta-1)}}$

O coeficiente de determinação, ou grau de previsibilidade para cada caráter $\left(\mathrm{R}^{2}\right)$, foi obtido por meio da predição do valor real do clone pela média de $\eta$ medidas repetidas. A precisão do procedimento será:

$$
\mathrm{R}^{2}=\rho_{\infty}^{2}=\frac{\eta \hat{\rho}}{1+\hat{\rho}(\eta-1)}
$$

em que $\hat{\rho}$ é o coeficiente de repetibilidade.

A predição do valor verdadeiro de cada clone (PVVC) ou a capacidade mais provável de cada genótipo foi obtida pelo critério de Lush (1964), que é: $\hat{\mathrm{Y}}_{\mathrm{ri}}=\mathrm{R}^{2} \overline{\mathrm{Y}}_{\mathrm{i}}+\left(1-\mathrm{R}^{2}\right) \overline{\mathrm{Y}}$, em que $\hat{\mathrm{Y}}_{\mathrm{ri}}$ é o estimador do valor real do i-ésimo clone; $\overline{\mathrm{Y}}_{\mathrm{i}}$ é a média do i-ésimo clone obtida a partir de $\eta$ avaliações; $\bar{Y}$ é a média geral da população, incluindo todos os i-ésimos clones e as $\eta$ avaliações.

\section{Resultados e Discussão}

Houve significância pelo teste $\mathrm{F}$ a $1 \%$ de probabilidade tanto entre cortes quanto entre clones, detectando a presença de variabilidade genética para todas as características (Tabela 1). Esta variabilidade reflete a heterogeneidade do material genético estudado, indicando a possibilidade de identificação de materiais promissores. O coeficiente de variação (CV) de $1,49 \%$ a $11,81 \%$ evidencia boa precisão experimental e, conseqüentemente, mostrou-se eficiente nas determinações

Tabela 1. Quadrados médios da análise da variância, médias do ensaio, coeficientes de variação, estimativas dos componentes genéticos e dos coeficientes de repetibilidade de características agroindustriais de genótipos de cana-de-açúcar, obtidos pelos métodos da análise de variância (MAV) e dos componentes principais (MCP).

\begin{tabular}{|c|c|c|c|c|c|c|}
\hline \multirow[t]{2}{*}{ Fonte de variação } & \multirow[t]{2}{*}{ GL } & \multicolumn{5}{|c|}{ Quadrado médio } \\
\hline & & Fibra & Pureza & $\mathrm{PCC}^{(1)}$ & $\mathrm{TCH}^{(2)}$ & $\mathrm{TPH}^{(3)}$ \\
\hline Cortes & 2 & $22,83 * *$ & $68,79 * *$ & $48,60 * *$ & $2.722,34 * *$ & $112,92 * *$ \\
\hline Genótipos & 19 & $2,28 * *$ & $4,01 * *$ & $1,07 * *$ & $471,68 * *$ & $10,30 * *$ \\
\hline Resíduo & 38 & 0,39 & 1,71 & 0,31 & 86,46 & 2,83 \\
\hline Médias do ensaio & & 12,74 & 87,65 & 14,60 & 97,40 & 14,25 \\
\hline Coeficiente de variação (\%) & & 4,96 & 1,49 & 3,82 & 9,55 & 11,81 \\
\hline Componente genético & & 0,63 & 0,77 & 0,25 & 128,40 & 2,49 \\
\hline Repetibilidade $\left(\hat{\rho}_{\mathrm{av}}\right)$ & & 0,61 & 0,31 & 0,44 & 0,60 & 0,47 \\
\hline Repetibilidade $\left(\hat{\rho}_{\mathrm{cp}}\right)$ & & 0,67 & 0,31 & 0,45 & 0,64 & 0,49 \\
\hline
\end{tabular}

${ }^{(1)}$ Porcentagem de pol no caldo da cana $(\%) .{ }^{(2)}$ Toneladas de cana/ha. ${ }^{(3)}$ Toneladas de pol no caldo da cana/ha. **Significativo a $1 \%$ de probabilidade pelo teste F. 
das características fibra, pureza, porcentagem de pol no caldo da cana (PCC), e toneladas de cana por hectare (TCH) e aceitável para o caráter tonelada de pol no caldo da cana por hectare (TPH).

$\mathrm{O}$ coeficiente de repetibilidade em ambos os métodos apresentou-se acima de 0,5 , sendo maior para as características fibra e toneladas de cana por hectare (TCH). Tal característica indica regularidade entre as repetições, podendo-se, então, esperar que estas variáveis expressem um bom controle genético. Para Vencovsky (1973), coeficientes de repetibilidade altos podem ser empregados como parâmetros para medir a capacidade de repetição da expressão do caráter avaliado. Por outro lado, pureza, porcentagem de pol no caldo da cana (PCC) e tonelada de pol (sacarose) no caldo da cana por hectare (TPH) exibiram valores abaixo de 0,5 , evidenciando que estes são muito influenciados pelo ambiente, ou seja, não houve regularidade na repetição dos caracteres de um corte para outro, conforme Cruz \& Regazzi (1997).

Apesar de existir uma pequena diferença das estimativas dos coeficientes de repetibilidade entre os dois métodos, não houve variação para a característica pureza. A esse respeito, Cruz \& Regazzi (1997) ressaltam que o método dos componentes principais é mais eficiente que o método da análise da variância, principalmente para as características que apresentam comportamentos cíclicos, como as demais características avaliadas.

Os coeficientes de determinação para as cinco características avaliadas, nas condições de estudo, demons- traram confiabilidade apenas para a fibra e toneladas de cana por hectare (TCH), que exibiram nos três cortes valores superiores a $80 \%$. As demais características ficaram abaixo deste valor, havendo necessidade de serem realizadas novas avaliações com um número de cortes bem maior (Tabela 2). Utilizando-se ambos os métodos, com cinco cortes, obtiveram-se valores acima de $80 \%$ para as características fibra, porcentagem de pol no caldo da cana (PCC), toneladas de cana por hectare (TCH) e toneladas de pol no caldo da cana por hectare (TPH), chegando até pouco mais que $90 \%$ para fibra pelo método dos componentes principais. Não é viável economicamente efetuar mais que cinco cortes para avaliações das características agroindustriais, tendo em vista que o aumento na precisão é muito pouco.

Quanto à capacidade mais provável de cada genótipo para cada característica em estudo, pelos dois métodos, 12 genótipos mostram-se acima da média dos padrões do Programa de Melhoramento Genético de Cana-deaçúcar (PMGCA) para fibra; seis para pureza; cinco para porcentagem de pol no caldo da cana (PCC); seis para toneladas de cana por hectare (TCH) e cinco para toneladas de pol no caldo da cana por hectare (TPH) (Tabela 3).

Pode-se selecionar para cada característica, os clones de maior interesse. Adotando-se um porcentual de seleção de $20 \%$ para a característica toneladas de pol (sacarose) no caldo da cana por hectare (TPH), seriam selecionados os clones RB9371, 16,35; RB9350, 15,69 e RB9364, 15,38; todos eles superiores aos padrões do Programa de Melhoramento Genético de Cana-de-açú-

Tabela 2. Características agroindustriais de clones em cana-de-açúcar, determinados pelo método da análise de variância (MAV) e pelo método dos componentes principais (MCP).

\begin{tabular}{|c|c|c|c|c|c|c|c|c|c|c|}
\hline \multirow[t]{2}{*}{ Número de cortes } & \multicolumn{2}{|c|}{ Fibra } & \multicolumn{2}{|c|}{ Pureza } & \multicolumn{2}{|c|}{$\mathrm{PCC}^{(1)}$} & \multicolumn{2}{|c|}{$\mathrm{TCH}^{(2)}$} & \multicolumn{2}{|c|}{$\mathrm{TPH}^{(3)}$} \\
\hline & MAV & MCP & MAV & $\mathrm{MCP}$ & MAV & $\overline{\mathrm{MCP}}$ & MAV & $\mathrm{MCP}$ & MAV & $\mathrm{MCP}$ \\
\hline 1 & 61,11 & 67,00 & 30,95 & 31,04 & 44,58 & 45,33 & 59,76 & 63,94 & 46,77 & 48,60 \\
\hline 2 & 75,86 & 80,24 & 47,27 & 47,38 & 61,67 & 62,38 & 74,81 & 78,00 & 63,73 & 65,41 \\
\hline 3 & 82,50 & 85,90 & 57,35 & 57,45 & 70,70 & 71,32 & 81,67 & 84,18 & 72,49 & 73,94 \\
\hline 4 & 86,27 & 89,04 & 64,19 & 64,29 & 76,29 & 76,83 & 85,59 & 87,64 & 77,85 & 79,09 \\
\hline 5 & 88,71 & 91,03 & 69,15 & 69,24 & 80,09 & 80,56 & 88,13 & 89,86 & 81,46 & 82,54 \\
\hline 10 & 94,02 & 95,31 & 81,76 & 81,82 & 88,94 & 89,24 & 93,69 & 94,66 & 89,78 & 90,44 \\
\hline 20 & 96,92 & 97,60 & 89,96 & 90,00 & 94,15 & 94,31 & 96,74 & 97,26 & 94,61 & 94,98 \\
\hline 50 & 98,74 & 99,02 & 95,73 & 95,75 & 97,57 & 97,64 & 98,67 & 98,88 & 97,77 & 97,93 \\
\hline 100 & 99,37 & 99,51 & 97,82 & 97,83 & 98,77 & 98,81 & 99,33 & 99,44 & 98,87 & 98,95 \\
\hline
\end{tabular}

(1)Porcentagem de pol no caldo da cana. ${ }^{(2)}$ Toneladas de cana/ha. ${ }^{(3)}$ Toneladas de pol no caldo da cana/ha. 
Tabela 3. Capacidade mais provável de cada genótipo quanto às características agroindustriais em cana-de-açúcar, determinadas pelo método da análise de variância (MAV) e pelo método dos componentes principais (MCP).

\begin{tabular}{|c|c|c|c|c|c|c|c|c|c|c|}
\hline \multirow[t]{2}{*}{ Clone } & \multicolumn{2}{|c|}{ Fibra } & \multicolumn{2}{|c|}{ Pureza } & \multicolumn{2}{|c|}{$\mathrm{PCC}^{(1)}$} & \multicolumn{2}{|c|}{$\mathrm{TCH}^{(2)}$} & \multicolumn{2}{|c|}{$\mathrm{TPH}^{(3)}$} \\
\hline & MAV & $\mathrm{MCP}$ & MAV & $\mathrm{MCP}$ & MAV & $\mathrm{MCP}$ & MAV & $\mathrm{MCP}$ & MAV & $\mathrm{MCP}$ \\
\hline RB72454 & 12,14 & 12,11 & 87,57 & 87,57 & 14,68 & 14,68 & 98,34 & 98,37 & 14,36 & 14,36 \\
\hline RB83102 & 12,40 & 12,38 & 87,76 & 87,76 & 14,73 & 14,73 & 103,25 & 103,43 & 15,16 & 15,18 \\
\hline SP791011 & 12,63 & 12,63 & 88,16 & 88,16 & 15,06 & 15,06 & 101,86 & 102,00 & 15,30 & 15,32 \\
\hline RB937 & 12,66 & 12,65 & 87,54 & 87,54 & 14,31 & 14,31 & 85,45 & 85,09 & 12,48 & 12,45 \\
\hline RB9318 & 13,77 & 13,81 & 87,98 & 87,99 & 14,25 & 14,25 & 97,20 & 97,19 & 13,79 & 13,78 \\
\hline RB9326 & 12,29 & 12,27 & 87,58 & 87,58 & 14,42 & 14,42 & 87,84 & 87,54 & 12,88 & 12,85 \\
\hline RB9331 & 12,43 & 12,42 & 88,18 & 88,18 & 14,96 & 14,96 & 99,67 & 99,74 & 14,91 & 14,92 \\
\hline RB9333 & 13,48 & 13,51 & 88,73 & 88,73 & 15,04 & 15,05 & 95,62 & 95,56 & 14,39 & 14,39 \\
\hline RB9340 & 12,43 & 12,41 & 88,20 & 88,20 & 14,70 & 14,70 & 103,88 & 104,08 & 15,18 & 15,20 \\
\hline RB9348 & 13,12 & 13,13 & 85,78 & 85,78 & 13,72 & 13,71 & 93,18 & 93,05 & 12,85 & 12,83 \\
\hline RB9350 & 11,88 & 11,84 & 87,52 & 87,52 & 15,21 & 15,21 & 102,85 & 103,02 & 15,66 & 15,69 \\
\hline RB9353 & 13,55 & 13,58 & 86,98 & 86,98 & 13,93 & 13,92 & 107,88 & 108,21 & 14,80 & 14,81 \\
\hline RB9354 & 11,27 & 11,21 & 88,40 & 88,41 & 14,87 & 14,87 & 73,67 & 72,94 & 11,39 & 11,33 \\
\hline RB9358 & 12,17 & 12,15 & 87,21 & 87,21 & 14,40 & 14,39 & 100,76 & 100,86 & 14,51 & 14,52 \\
\hline RB9362 & 12,56 & 12,55 & 87,22 & 87,22 & 14,61 & 14,61 & 99,32 & 99,38 & 14,62 & 14,62 \\
\hline RB9364 & 14,02 & 14,08 & 87,42 & 87,42 & 14,36 & 14,36 & 108,55 & 108,89 & 15,36 & 15,38 \\
\hline RB9366 & 12,88 & 12,89 & 86,96 & 86,96 & 14,18 & 14,18 & 107,09 & 107,38 & 15,06 & 15,07 \\
\hline RB9367 & 12,12 & 12,09 & 88,13 & 88,14 & 15,38 & 15,38 & 92,18 & 92,02 & 14,43 & 14,43 \\
\hline RB9371 & 13,67 & 13,71 & 88,33 & 88,33 & 14,71 & 14,71 & 112,88 & 113,36 & 16,31 & 16,35 \\
\hline RB9379 & 13,34 & 13,36 & 87,39 & 87,39 & 14,52 & 14,52 & 76,61 & 75,97 & 11,60 & 11,55 \\
\hline $\begin{array}{l}\text { Médias dos padrões } \\
\text { do PMGCA }{ }^{(4)}\end{array}$ & & & & & & & & & & \\
\hline
\end{tabular}
do $\mathrm{PMGCA}^{(4)}$

${ }^{(1)}$ Porcentagem de pol no caldo da cana (\%). ${ }^{(2)}$ Toneladas de cana/ha. ${ }^{(3)}$ Toneladas de pol no caldo da cana/ha. ${ }^{(4)}$ Programa de Melhoramento Genético de Cana-de-açúcar.

car (PMGCA), e com previsibilidade de produção $73,94 \%$. No entanto, esta previsibilidade é intermediária, havendo a necessidade de se considerar pelo menos cinco cortes. Considerando a produção agrícola toneladas de cana por hectare (TCH), seriam selecionados os clones RB9371, 113,86; RB9364, 108,89; e RB9353, 108,21 , com previsibilidade de $84,18 \%$ a partir de três cortes.

\section{Conclusões}

1. As estimativas de repetibilidade acima de 0,5 para fibra e tonelada de cana por hectare são regulares com confiabilidade acima de $84 \%$, considerando-se o método dos componentes principais.

2. As baixas estimativas de repetibilidade para pureza, porcentagem de pol no caldo da cana e tonelada de pol no caldo da cana por hectare, com previsibilidade abaixo de $74 \%$, indicam que não há regularidade na repetição dessas variáveis de um corte para outro e que um maior número de avaliações é necessário.
3. Os métodos dos componentes principais e análise de variância evidenciaram, em cinco cortes, uma previsibilidade maior que $80 \%$ para fibra, porcentagem de pol no caldo da cana, tonelada de cana por hectare e tonelada de pol no caldo da cana por hectare, sendo mais eficiente pelo primeiro método.

4. Os clones RB9371, RB9350 e RB9364 são os mais promissores para as características tonelada de cana por hectare e tonelada de pol no caldo da cana por hectare e poderão ser indicados para o cultivo após avaliações em outros ambientes.

\section{Agradecimentos}

À Capes e à Fapeal, pela concessão de bolsas a Maria Silvia Monteiro dos Santos e José Antônio Madalena; aos técnicos e empregados rurais das unidades onde foram conduzidos os experimentos, pelo empenho e dedicação; ao Dep. de Fitotecnia e Fitossanidade da Universidade Federal de Alagoas e ao Programa de Melhoramento Genético da Cana-de-açúcar (PMGCA). 


\section{Referências}

ABEYWARDENA, V. An application of principal component analysis in genetics. Journal of Genetics, v.16, p.27-51, 1972.

BARBOSA, G.V.S.; SOUZA, A.J.R.; ROCHA, A.M.C.; RIBEIRO, C.A.G.; FERREIRA, J.L.C.; SOARES, L.; CRUZ, M.M.; SILVA, W.C.M. Novas variedades RB de cana-de-açúcar para Alagoas. Maceió: UFAL; Programa de Melhoramento Genético de Cana-deAçúcar, 2000. 16p. (Boletim Técnico Programa de Melhoramento Genético de Cana-de-Açúcar, 1).

BOTREL, M.A.; FERREIRA, R.P.; CRUZ, C.D.; PEREIRA, A.V.; VIANA, M.C.M.; ROCHA, R.; MIRANDA, M. Estimativas de coeficientes de repetibilidade para produção de matéria seca em cultivar de alfafa, sob diferentes ambientes. Revista Ceres, v.47, p.651-663, 2000.

COSTA, J.G.; LEDO, A.S.; OLIVEIRA, M.N. Estimativas de repetibilidade de características de frutos de cupuaçuzeiro no Estado do Acre. Revista Brasileira de Fruticultura, v.19, p.313-318, 1997.

CRUZ, C.D.; REGAZZI, A.J. Modelos biométricos aplicados ao melhoramento genético. Viçosa: UFV, 1994. 394p.

CRUZ, C.D.; REGAZZI, A.J. Modelos biométricos aplicados ao melhoramento genético. 2.ed. Viçosa: UFV, 1997. 390p.

FALCONER, D.S. Introdução à genética quantitativa. Viçosa: UFV, 1987. 279p.

FONSECA, C.E.L.; ESCOBAR, J.R.; BUENO, D.M. Variabilidade de alguns caracteres físicos e químicos do fruto do cupuaçuzeiro. Pesquisa Agropecuária Brasileira, v.25, p.1079-1084, 1990.
GONÇALVES, P.S.; CARDOSO, M.; SÁES, L.A. Estimativas de repetibilidade na seleção de árvores adultas de seringueira. Pesquisa Agropecuária Brasileira, v.25, p.1031-1038, 1990.

GONÇALVES, P.S.; MARTINS, A.L.M.; BORTOLETTO, N.; UTIDA, R.C. Variabilidade dos caracteres estruturais do sistema laticífero da casca em plantas jovens de seringueira. Pesquisa Agropecuária Brasileira, v.30, p.649-656, 1995.

GONÇALVES, P.S.; ROSSETTI, A.G.; PAIVA, J.R. Coeficiente de repetibilidade e eficiência do miniteste de produção na seleção de plantas de seringueira. Pesquisa Agropecuária Brasileira, v.17, p.233-237, 1982.

LUSH, J.L. Melhoramento genético dos animais domésticos. Tradução de G.G. Carneiro, J.M.P. Memoria, G. Drummond. Rio de Janeiro: USAID, 1964. 570p.

MAGALHÃES, D.S. Eficiência de diferentes implementos relacionados ao teor de umidade do solo na destruição da socaria de cana-de-açúcar. 2000. 18p. Monografia (Graduação) Universidade Federal de Alagoas, Rio Largo.

MANSOUR, H.; NORDHEIM, E.V.; RULEDGE, J.J. Estimators of repeatability. Theoretical and Applied Genetics, v.60, p.151$156,1981$.

RUTLEDGE, J.J. A scaling which remove bias of Abeywardena's estimator of repeatability. Journal of Genetics, v.61, p.247-250, 1974.

SIQUEIRA, E.R. Coeficiente de repetibilidade da produção de frutos do coqueiro comum. Pesquisa Agropecuária Brasileira, v.17, p.573-574, 1982,

VENCOVSKY, R. Princípios de genética quantitativa. Piracicaba: Esalq, 1973. 97p.

Recebido em 14 de março de 2003 e aprovado em 22 de dezembro de 2003 